\title{
Computer-Aided Drug Potency Decoder: A Biomedical/Bioengineering Device for Determining and Distinguishing Drug Efficacies
}

\section{Norbert Nwankwo*}

Department of Clinical Pharmacy, Faculty of Pharmacy, University of Port Harcourt, Port Harcourt, Rivers State, Nigeria

\begin{abstract}
We have preliminarily presented three Digital Signal Processing (DSP)-based Computer-Aided Biomedical/ Bioengineering devices. They are Computer-Aided Drug Resistance Calculator (Patent Application Publication No: US20150370964), Computer-Aided Vaccine Potency Assessor (InnoCentive (USA) Challenge Winning Solver Award ID: 9933477) and Computer-Aided Pharmaco-Investigator.
\end{abstract}

Here, we present the fourth biomedical/bioengineering device, which is to be labeled Computer-Aided Drug Potency Decoder. Its role is to determine and distinguish drug effectiveness.

All the DSP-based devices including those mentioned here engaged aggregate contributions from all the Molecular descriptors and point mutations. This is in order to obtain the entirety of the pharmacological activities involved. It is therefore vital that all the Molecular descriptors and point mutations need be identified and engaged. This is to ensure that accurate pharmacological activities are obtained. Engagement of incomplete and incorrect Molecular descriptors and point mutations will lead to deriving incorrect pharmacological activities. Engaging false results hence inaccurate pharmacological activities portend danger to the end-users (patients). There are no known procedures or algorithms to determine complete and accurate Molecular descriptors and point mutations for use in the DSP-based ComputerAided devices. However, the researcher has developed a procedure that engages all Molecular descriptors and point mutations, and presents accurate findings. This procedure needs be incorporated into all the devices.

Keywords: Computer-Aided Pharmaco-Investigator; Biomedical; Bioengineering; HIV

\section{Introduction}

Earlier, we have developed three Digital Signal Processing (DSP)based Computer-Aided Biomedical/Bioengineering devices [1]. They include Computer-Aided Drug Resistance Calculator (Patent Application Publication No: US20150370964) and Computer-Aided Vaccine Potency Assessor. The Assessor won the InnoCentive (USA) Challenge Winning Solver Award (ID: 9933477). The third is the Computer-Aided Pharmaco-Investigator [1].

Computer-Aided Drug Resistance Calculator (if patent is granted) is envisaged to rationally assess resistances acquired by organisms and viruses exposed to various drugs in the course of treatment [1]. This is in order to provide adequate choices that will grant the required therapeutic intervention. Computer-Aided Vaccine Potency Assessor is expected to be developed by InnoCentive with the view of providing manufacturers of vaccines with a computerized resource-, labor- and time-saving biomedical/bioengineering device that will assist in evaluating and comparing efficacies of their already produced vaccines or those being designed. In the course of the award, we agreed that InnoCentive should develop it further. The third device, Computer-Aided Pharmaco-Investigator is envisioned to provide firms (pharmaceutical, biotechnological, etc), universities, research institutions, etc with a simpler and cheaper approach to investigating characteristics that are presented in the protein sequence information arising from interactions with their bio-active substances. The substances could, in nature, be protein/peptide, protein targets or genes/proteins encoding the bio-active substances.

Here, we present the fourth biomedical/bioengineering device, which is to be labeled Computer-Aided Drug Potency Decoder. This device will be engaged in determining and distinguishing drug effectiveness. It has initially been demonstrated [2-4]. Informational
Spectrum Method (ISM), a technique, which provided for the three other biomedical/bioengineering devices, is engaged. ComputerAided Drug Potency Decoder also engages sequence information. In demonstrating the workability of the Computer-Aided Vaccine Potency Assessor for InnoCentive, ISM technique and the sequence information of two Plasmodial vaccine candidates (P18 and P32) were employed. Similarly, ISM technique and the sequence information of two protein-based anti-retroviral agents (Enfuvirtide and Sifuvirtide) and their target protein (N-terminal of the Hepad Repeat (NHR or HR1), are engaged in elucidating the practicability of the ComputerAided Drug Potency Decoder [2-4].

Though the functioning of the Computer-Aided Drug Potency Decoder has been discussed [2-4], and the technique detailed [2-16] a concise description of the running of the device is briefly provided here. The initial step in the procedure is to translate the alphabetic codes of the protein sequences (e.g. Enfuvirtide and Sifuvirtide and NHR) into numbers by engaging the corresponding values of the Molecular descriptors (Amino Acid Indices or Biological Parameters) involved. This transforms the protein-based bio-active substances or their protein targets or genes/protein encoding the bio-active substances into signals (numerical sequences). The signals are then analyzed using

*Corresponding author: Norbert Nwankwo, Department of Clinical Pharmacy, Faculty of Pharmacy, University of Port Harcourt, Port Harcourt, Rivers State, Nigeria, Tel: +2348065827014; E-mail: nobatn@gmail.com

Received: October 07, 2015; Accepted: December 14, 2015; Published: December 31,2015

Citation: Nwankwo N (2015) Computer-Aided Drug Potency Decoder: A Biomedical/Bioengineering Device for Determining and Distinguishing Drug Efficacies. J Bioengineer \& Biomedical Sci 6: 176. doi:10.4172/2155-9538.1000176

Copyright: @ 2015 Nwankwo N. This is an open-access article distributed under the terms of the Creative Commons Attribution License, which permits unrestricted use, distribution, and reproduction in any medium, provided the original author and source are credited. 
Discrete Fourier Transform (DFT) and all the mutations (sequence information) as well as Molecular descriptors. This is in order to uncover and present in numerical terms, the pharmacological behavior of the bio-active substances. The behavior presented by all the amino acid changes and Molecular descriptors are then aggregated to account for the entire pharmacological activities. This information is then utilized to determine and distinguish drug efficacies.

In order to identify the sequence information and Molecular Descriptors engaged, interaction between the two anti-retroviral agents (Enfuvirtide and Sifuvirtide) and their target protein NHR were first investigated at the atomic level. Initial investigations have shown that helical and hydrophobic-based interactions between the $\mathrm{N}$-terminal of the Hepad Repeat (NHR or HR1) and the C-terminal of the Hepad Repeat (CHR or HR2) of the HIV Transmembrane protein (HIV gp41), and re-arrangement is inevitably required for HIV infection to proceed to AIDS disease [17]. An analogue of the CHR called Enfuvirtide (already approved by FDA) has been designed and employed effectively in managing HIV/AIDS [18]. By means of bio-engineering tinkering, another CHR analogue, Sifuvirtide was later designed [18]. Using clinical, CD Spectrometric and Biophysical experiments, Sifuvirtide was observed to be more efficacious than the Enfuvirtide though it is still being tried clinically for use in managing HIV/AIDS [18].

The bio-engineering modifications were acknowledged to have engaged a total of eight helicity- and hydrphobicity-based Molecular Descriptors and four sequence alterations [2-4]. According to the study [18], the modifications were achieved through the introduction of the charged amino acids (Glutamic acid and Serine); provision of hydrophobic pocket by replacing Glutamic acid at position 119 by Threonine (E119T); and an addition of Serine to the N-terminus so as to enhance stability. The end-result of the bioengineering alterations that gave birth to Sifuvirtide is a change in the helicity and hydrphobicity. Using the Computer-Aided Drug Potency Decoder, the calculated potencies, of the Enfuvirtide (72.33\%) and Sifuvirtide $(85.42 \%)$ disclosed that Sifuvirtide is more potent. The target protein, NHR provided $87.07 \%$ availability to interact with the agents [24]. Our finding that Sifuvirtide is more potent than Enfuvirtide is in accord with those made with the other procedures (CD Spectroscopic, Biophysical and clinical laboratory experiments).

In order to obtain the total pharmacological activities in the bio-active substances, DSP-based procedure engages aggregate contributions made by all the mutations and Molecular Descriptors. This includes all Computer-Aided Biomedical/Bioengineering devices developed. This is because it has been recognized that at one point mutation, more than one Molecular descriptor is involved [19]. As a result, all Molecular Descriptors and mutations must be detected and utilized. On the other hand, engaging incomplete and incorrect mutations and Molecular Descriptors will lead to erroneous findings. In order to uncover all the Molecular Descriptors and mutations, the mechanisms of the interactions involved must be thorough investigated at the atomic level. Unfortunately, this is impossible. This is because there could be some mechanisms of interactions that are not yet identified. There could also be some mechanism of interactions that have not been acknowledged in the literature.

To develop reliable Computer-Aided Biomedical/Bioengineering devices, which are fit to be engaged in human (or even animals), devices that will engage complete and exact mutations and Molecular Descriptors, hence render accurate results, must be employed. Contrarily, false results will be obtained. Bio-medical and bio- engineering devices that produce false results portend danger for the healthcare delivery system. This is because falsely assessed potencies of drug, vaccines drug resistance and other pharmacological properties pose a terrible risk to the user/patients.

Based on these, there is need to develop an approach/procedure (mathematical model, program, etc) that would identify appropriate and complete mutations and parameters. Apart from the bio-medical and bio-engineering device innovated; the researcher has also developed a procedure that engages complete and exact mutations and Molecular Descriptors.

In our earlier Research News Article titled "Pharmacists, Manufacturers of Pharmaceuticals, Clinicians, Scientists, Researchers and Environmentalists can now carry out their normal functions without engaging reagents and equipments but Computers only" and publication [3], we maintained that rational and computerized pharmacological assessment is currently complementing the clinical approaches and may take over in future [20]. Here, we present the fourth bio-medical and bio-engineering device that will assist healthcare professionals in accomplishing their tasks.

\section{References}

1. Nwankwo N (2015) Computer-Aided Pharmaco-Investigator: A Biomedical/ Bioengineering Device for Pharmacological Investigations. Austin Virol and Retrovirology 2(2): 1015

2. Nwankwo Norbert, Godwin Molokwu, Ngozika Njoku (2015) "Nove Computerized Approaches to Investigating Pharmacological Activities". Computational Biology and Bioinformatics 3: 52-64.

3. Nwankwo N (2012) "Signal processing-based Bioinformatics methods for characterization and identification of Bio-functionalities of proteins", PhD Thesis (accepted), De Montfort University, Leicester, United Kingdom.

4. Nwankwo N, Seker H(2011) "Digital Signal Processing Techniques: Calculating the Biological Functionalities of Proteins" J Proteomics Bioinform 4: 260-268.

5. Nwankwo N (2015) Can Bio-functionalities be deciphered from Protein Sequence Information using Computational Approaches? 29th Annual Symposium of the Protein Society Book Abstract: 407. 2015.

6. Nwankwo N (2015) Direct Computerized Translation of Biological data into Biological Information is now feasible: the Gains of Digital Signal Processingbased Bioinformatics Techniques. Virol-mycol 4: 52.

7. Veljković V, Cosić I, Dimitrijević B, Lalović D (1985) Is it possible to analyze DNA and protein sequences by the methods of digital signal processing? IEEE Trans Biomed Eng 32: 337-341.

8. Veljkovic V, Veljkovic N (2009) Characterization of conserved properties of hemagglutinin of $\mathrm{h} 5 \mathrm{~h} 1$ and human influenza viruses: possible consequences for therapy and infection control. BMC Structural Biology 9: 1-11.

9. Veljkovic V, Glisic S, Veljkovic N, Bojic T, Dietrich U, et al. (2014) Influenza vaccine as prevention for cardiovascular diseases: possible molecular mechanism. Vaccine 32: 6569-6575.

10. Nwankwo N (2013) "A Digital Signal Processing-based Bioinformatics Approach to Identifying the Origins of HIV-1 non B subtypes infecting US Army Personnel serving abroad". Curr HIV Res 11: 271-280.

11. Doliana R, Veljkovic V, Prljic J, Veljkovic N, De Lorenzo E, et al. (2008) EMILINs interact with anthrax protective antigen and inhibit toxin action in vitro. Matrix Biol 27: 96-106.

12. Veljkovic V, Glisic S, Veljkovic N, Jovanovic Cupic S, Prljic J, et al. (2014) Assessment of Hepatitis $C$ Virus protein sequences with regard to interferon ribavirin combination therapy response in patients with HCV genotype $1 \mathrm{~b}$. Vaccine 32: 6569-6575.

13. Cosic I (1994) Macromolecular bioactivity: is it resonant interaction between macromolecules?--Theory and applications. IEEE Trans Biomed Eng 41: 1101-1114.

14. Nwankwo N, Seker H (2013) "HIV Progression to AIDS: Bioinformatics Approach to Determining the Mechanism of Action". Curr HIV Res 11: 30-42.

15. Nwankwo N, Seker H (2010) "A signal processing-based bioinformatics 
Citation: Nwankwo N (2015) Computer-Aided Drug Potency Decoder: A Biomedical/Bioengineering Device for Determining and Distinguishing Drug Efficacies. J Bioengineer \& Biomedical Sci 6: 176. doi:10.4172/2155- 9538.1000176

Page 3 of 3

approach to assessing drug resistance: human immunodeficiency virus as a case study". Conf Proc IEEE Eng Med Biol Soc 2010: 1836-1839.

16. Nwankwo N, Adikwu M, Okafor I (2015) “HIV Tropism Prediction: Digital Signal Processing-Based Bioinformatics Approach is Non-Sequence Alignment Dependent". Computational Biology and Bioinformatics 3: 21-30.
17. Menendez-Arias L (2010) "Molecular basis of Human Immunodeficiency Virus drug resistance: an update". Antiviral Res 85: 210-231.

18. He Y, Xiao Y, Song H, Liang Q, Ju D, et al. (2008) Design and evaluation of sifuvirtide, a novel hiv-1 fusion inhibitor". J Biol Chem 283: 11126-11134.

19. Hoj L (2008) "In silico identification of physiochemical properties at mutating position positions relevant to reducing susceptibility to Amprenavir", XVII International HIV Drug Resistance Workshop, Poster No.113, 2008. 